\title{
Approximate natural vibration analysis of rectangular plates with openings using assumed mode method
}

\author{
Dae Seung Cho ${ }^{1}$, Nikola Vladimir ${ }^{2}$ and Tae Muk $\mathrm{Choi}^{3}$ \\ ${ }^{1}$ Department of Naval Architecture and Ocean Engineering, Pusan National University, Busan, Korea \\ ${ }^{2}$ University of Zagreb, Faculty of Mechanical Engineering and Naval Architecture, Zagreb, Croatia \\ ${ }^{3}$ Createch Co. Ltd., Busan, Korea
}

\begin{abstract}
Natural vibration analysis of plates with openings of different shape represents an important issue in naval architecture and ocean engineering applications. In this paper, a procedure for vibration analysis of plates with openings and arbitrary edge constraints is presented. It is based on the assumed mode method, where natural frequencies and modes are determined by solving an eigenvalue problem of a multi-degree-of-freedom system matrix equation derived by using Lagrange's equations of motion. The presented solution represents an extension of a procedure for natural vibration analysis of rectangular plates without openings, which has been recently presented in the literature. The effect of an opening is taken into account in an intuitive way, i.e. by subtracting its energy from the total plate energy without opening. Illustrative numerical examples include dynamic analysis of rectangular plates with rectangular, elliptic, circular as well as oval openings with various plate thicknesses and different combinations of boundary conditions. The results are compared with those obtained by the finite element method (FEM) as well as those available in the relevant literature, and very good agreement is achieved.
\end{abstract}

KEY WORDS: Natural vibration analysis; Flexural vibrations; Assumed mode method; Lagrange's equations; Rectangular plate; Plate opening; Arbitrary boundary constraints.

\section{INTRODUCTION}

Dynamic analysis of plates with openings represents an important issue in naval architecture and civil, mechanical as well as ocean engineering. Problem of determining flexural natural frequencies of plates with central free openings has been studied by many researchers (Leissa, 1969; Monahan et al., 1970; Bathe et al., 1973; Paramasivam, 1973; Hegarty and Ariman, 1975; Ali and Atwal, 1980; Laura et al., 1987; Gutierrez et al., 1987). Nowadays, the finite element method gives complete solution to a problem of vibration of plates with openings, and although it applied for very long time (Monahan et al., 1970), it still suffers from the computational time consumption. Finite difference method has been applied to a plate with opening and simply supported and clamped edges by Paramasivam (1973). Reddy (1982) analysed large amplitude flexural vibrations of layered composite plates with openings. Grossi et al. (1997) applied optimized Rayleigh-Ritz method to generate values of the fundamental frequency coefficient corresponding to the first fully antisymmetric mode for rectangular plates with circular holes and elastically restrained against rotation. In all these references positions of the openings are limited to plate central part. However, finite difference method has been applied to a plate with more than two openings by Aksu and Ali (1976). Huang and Sakiyama (1999) proposed approximate method for free vibration analysis of rectangular plates with openings of different shapes (circular,

Corresponding author: Dae Seung Cho

e-mail:daecho@pusan.ac.kr 
semi-circular, elliptic, square, rectangular, triangular, etc.). They consider a plate with an opening as a body with non-uniform thickness, where the opening is treated as an extremely thin part of the plate. In that sense the problem is translated into the free vibration analysis of the equivalent rectangular plate with non-uniform thickness by applying the discrete solution (Huang and Sakiyama, 1998). It should be noted that the problem is solved for arbitrary position of the opening within the plate area. An extensive literature survey on vibration analysis of rectangular plates with openings, with particular emphasis to application of Rayleigh-Ritz method, has been presented by Kwak and Han (2007). They proposed a Rayleigh-Ritz based method with different coordinate systems for a plate and opening with particular aim to simplify integration process in total energy determination.

Recently, assumed mode method using characteristic orthogonal polynomials is applied to problem of free vibration of rectangular plates of arbitrary edge constraints (Chung et al., 1993; Kim et al., 2012). In this paper, assumed mode method is applied to natural vibration analysis of plates with openings of different shape and arbitrary boundary conditions. The opening effect is accounted for by subtracting its strain and kinetic energies from corresponding total plate energies without openings. Natural frequencies and corresponding mode shapes are obtained by solving an eigenvalue problem of a multi-degree-offreedom system matrix equation, which has been derived by Lagrange's equations of motion. It should be noted also that the proposed method can be applied for arbitrary number of openings and their arbitrary positions. Numerical examples include analysis of natural vibrations of rectangular plates with rectangular, elliptic, circular and oval openings. Both, thin and thick plates are considered. The results are compared with those obtained by the finite element method as well as some results available in the relevant literature. It is shown that the presented procedure can be used as a reliable alternative to widely used FEM, which may require much more time in model preparation, as for instance in case of plate with oval opening.

\section{MATHEMATICAL MODEL}

\section{Equations of motion}

The Mindlin thick plate theory, which takes shear influence and rotary inertia into account, is introduced (Mindlin et al., 1956). In that way the method can be applied not only to thin plates, but also to moderately thick and thick ones. The Mindlin theory deals with three general displacements, i.e. plate deflection $w$, and angles of cross-section rotation about $x$ and $y$ axis, $\psi_{x}$ and $\psi_{y}$, respectively. From the equilibrium of sectional forces (bending moments, torsional moments and shear forces) and inertia forces, equations of motions are derived:

$$
\begin{gathered}
\frac{\rho h^{3}}{12} \frac{\partial^{2} \psi_{x}}{\partial t^{2}}-D\left(\frac{\partial^{2} \psi_{x}}{\partial x^{2}}+\frac{1}{2}(1-v) \frac{\partial^{2} \psi_{x}}{\partial y^{2}}+\frac{1}{2}(1+v) \frac{\partial^{2} \psi_{y}}{\partial x \partial y}\right)-k G h\left(\frac{\partial w}{\partial x}-\psi_{x}\right)=0 \\
\frac{\rho h^{3}}{12} \frac{\partial^{2} \psi_{y}}{\partial t^{2}}-D\left(\frac{\partial^{2} \psi_{y}}{\partial y^{2}}+\frac{1}{2}(1-v) \frac{\partial^{2} \psi_{y}}{\partial x^{2}}+\frac{1}{2}(1+v) \frac{\partial^{2} \psi_{x}}{\partial x \partial y}\right)-k G h\left(\frac{\partial w}{\partial y}-\psi_{y}\right)=0 \\
\frac{\rho}{k G} \frac{\partial^{2} w}{\partial t^{2}}-\frac{\partial^{2} w}{\partial x^{2}}-\frac{\partial^{2} w}{\partial y^{2}}+\frac{\partial \psi_{x}}{\partial x}+\frac{\partial \psi_{y}}{\partial y}=0
\end{gathered}
$$

where $\rho$ is plate density, $h$ is plate thickness, $k$ is shear coefficient, while $v$ is Poisson's ratio. Further on, $D$ represents plate flexural rigidity $D=E h^{3} /\left(12\left(1-v^{2}\right)\right)$, while $E$ and $G=E /(2(1+v))$ are Young's and shear modulus, respectively.

The basic idea to account for an opening effect is to subtract the energy of the opening part from the total plate energy without openings. In that sense, following expressions for the strain and kinetic energy of rectangular plate with openings, respectively, are valid: 


$$
\begin{aligned}
& V=V_{p}-V_{o}, \\
& T=T_{p}-T_{o} .
\end{aligned}
$$

where $V_{p}$ and $V_{o}$ are strain energies and $T_{p}$ and $T_{o}$ are kinetic energies of plate and opening, respectively.

\section{Energy of a rectangular plate without openings}

By introducing non-dimensional parameters $\xi=x / a, \eta=y / b, \alpha=a / b$ and $S=k G h / D$ for a rectangular plate of length $a$ and breadth $b$, one can write for the strain and kinetic energy of rectangular plate without openings, $V$ and $T$, respectively (Kim et al., 2012):

$$
\begin{aligned}
& V_{p}=\frac{D}{2 \alpha} \int_{0}^{1} \int_{0}^{1}\left[\left(\frac{\partial \psi_{\xi}}{\partial \xi}\right)^{2}+\alpha^{2}\left(\frac{\partial \psi_{\eta}}{\partial \eta}\right)^{2}+2 v \alpha \frac{\partial \psi_{\xi}}{\partial \xi} \frac{\partial \psi_{\eta}}{\partial \eta}+\frac{1-v}{2}\left(\alpha \frac{\partial \psi_{\xi}}{\partial \eta}+\frac{\partial \psi_{\eta}}{\partial \xi}\right)^{2}\right. \\
&\left.+S\left(\left(\frac{\partial w}{\partial \xi}-a \psi_{\xi}\right)^{2}+\alpha^{2}\left(\frac{\partial w}{\partial \eta}-b \psi_{\eta}\right)^{2}\right]\right] \mathrm{d} \xi \mathrm{d} \eta \\
&+\int_{0}^{1}\left[K_{R x 1} \psi_{\xi}^{2}(0, \eta)+S K_{T x 1} w^{2}(0, \eta)\right] \mathrm{d} \eta+\alpha^{2} \int_{0}^{1}\left[K_{R y 1} \psi_{\eta}^{2}(\xi, 0)+S K_{T y 1} w^{2}(\xi, 0)\right] \mathrm{d} \xi \\
&+\int_{0}^{1}\left[K_{R x 2} \psi_{\xi}^{2}(1, \eta)+S K_{T x 2} w^{2}(1, \eta)\right] \mathrm{d} \eta+\alpha^{2} \int_{0}^{1}\left[K_{R y 2} \psi_{\eta}^{2}(\xi, 1)+S K_{T y 2} w^{2}(\xi, 1)\right] \mathrm{d} \xi \\
& T_{p}=\frac{\rho a b}{2} \int_{0}^{1} \int_{0}^{1}\left[h\left(\frac{\partial w}{\partial t}\right)^{2}+\frac{h^{3}}{12}\left(\frac{\partial \psi_{\xi}}{\partial t}\right)^{2}+\frac{h^{3}}{12}\left(\frac{\partial \psi_{\eta}}{\partial t}\right)^{2}\right] \mathrm{d} \xi \mathrm{d} \eta
\end{aligned}
$$

where $K_{T x 1}=\left(k_{T x 1} a / k G h\right), K_{T x 2}=\left(k_{T x 2} a / k G h\right), K_{T y 1}=\left(k_{T y 1} b / k G h\right)$ and $K_{T y 2}=\left(k_{T y 2} b / k G h\right)$ are non-dimensional stiffness at $x=0, x=a, y=0$ and $y=b$, respectively, and correspond to the translational spring constants per unit length $k_{T x 1}, k_{T x 2}, k_{T y 1}$ and $k_{T y 2}$ shown in Fig. 1. In the same manner, $K_{R x 1}=\left(k_{R x 1} a / D\right), K_{R x 2}=\left(k_{R x 2} a / D\right)$, $K_{R y 1}=\left(k_{R y 1} b / D\right)$ and $K_{R y 2}=\left(k_{R y 2} b / D\right)$ are for the rotational spring constants per unit length $k_{R x 1}, k_{R x 2}, k_{R y 1}$ and $k_{R y 2}$ shown in Fig. 1, respectively. In Fig. 1 the following quantities are related to $x$ direction: $a, k_{T x 1}, k_{T x 2}, k_{R x 1}$ and $k_{R x 2}$. Other quantities $\left(b, k_{T y 1}, k_{T y 2} k_{R y 1}\right.$ and $\left.k_{R y 2}\right)$ are relevant for $y$ direction.

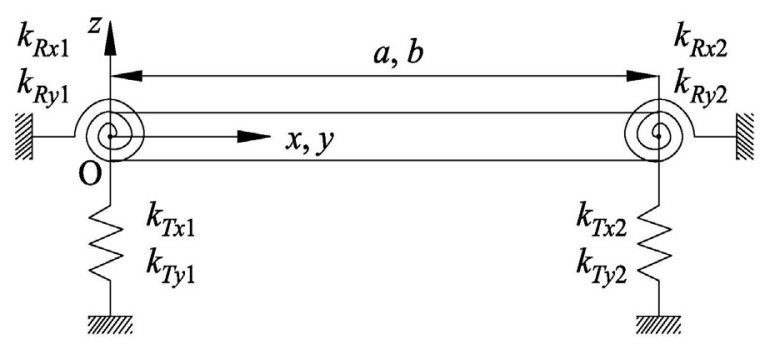

Fig. 1 Rectangular plate elastically supported in translational and rotational directions.

\section{Energy of rectangular opening}

In similar way, as for a plate without openings, one can write for strain and kinetic energy of rectangular opening shown in Fig. 2, respectively: 


$$
\begin{gathered}
V_{r o}=\frac{D}{2 \alpha} \int_{y_{r o}-b_{r o}}^{y_{r o}+b_{r o} x_{r o}-a_{r o}+a_{r o}}\left[\left(\frac{\partial \psi_{\xi}}{\partial \xi}\right)^{2}+\alpha^{2}\left(\frac{\partial \psi_{\eta}}{\partial \eta}\right)^{2}+2 v \alpha \frac{\partial \psi_{\xi}}{\partial \xi} \frac{\partial \psi_{\eta}}{\partial \eta}+\frac{1-v}{2}\left(\alpha \frac{\partial \psi_{\xi}}{\partial \eta}+\frac{\partial \psi_{\eta}}{\partial \xi}\right)^{2}\right. \\
\left.+S\left(\left(\frac{\partial w}{\partial \xi}-a \psi_{\xi}\right)^{2}+\alpha^{2}\left(\frac{\partial w}{\partial \eta}-b \psi_{\eta}\right)^{2}\right)\right] \mathrm{d} \xi \mathrm{d} \eta \\
T_{r o}=\frac{\rho a b}{2} \int_{y_{r o}-b_{r o}}^{y_{r o}+b_{r o} x_{r o}-a_{r o}+a_{r o}}\left[h\left(\frac{\partial w}{\partial t}\right)^{2}+\frac{h^{3}}{12}\left(\frac{\partial \psi_{\xi}}{\partial t}\right)^{2}+\frac{h^{3}}{12}\left(\frac{\partial \psi_{\eta}}{\partial t}\right)^{2}\right] \mathrm{d} \xi \mathrm{d} \eta,
\end{gathered}
$$

In Fig. $2 x_{r o}$ and $y_{r o}$ denote longitudinal and transverse coordinate of rectangular opening centre of gravity and $a_{r o}$ and $b_{r o}$ are one half of its length and breadth.
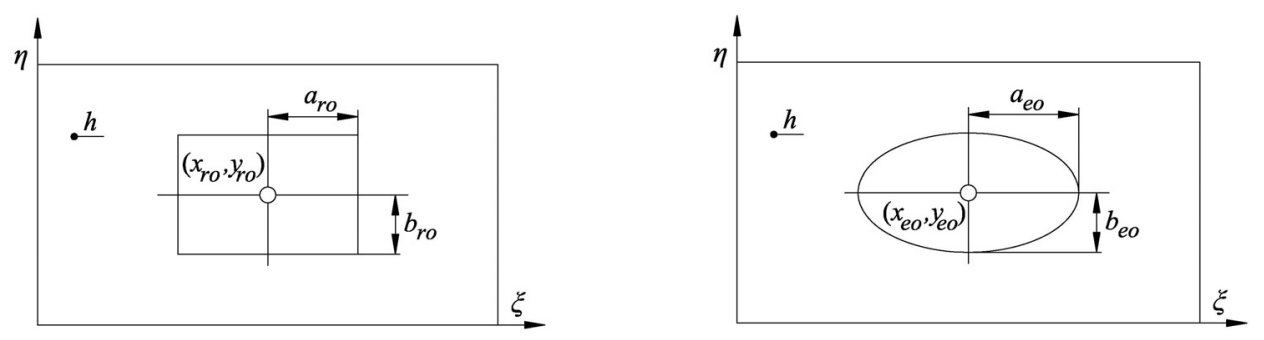

Fig. 2 Rectangular plate with rectangular opening. Fig. 3 Rectangular plate with elliptic opening.

\section{Energy of elliptic opening}

Analogously to the rectangular opening, strain and kinetic energies of elliptical opening shown in Fig. 3 yield:

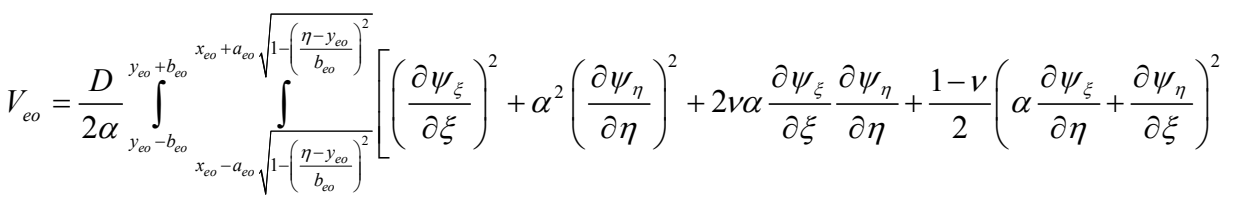

$$
\begin{aligned}
& \left.+S\left(\left(\frac{\partial w}{\partial \xi}-a \psi_{\xi}\right)^{2}+\alpha^{2}\left(\frac{\partial w}{\partial \eta}-b \psi_{\eta}\right)^{2}\right)\right] \mathrm{d} \xi \mathrm{d} \eta \\
& \left.T_{e o}=\frac{\rho a b}{2} \int_{y_{r o}-b_{r o}}^{y_{r o}+b_{r o}} \int_{x_{e o}-a_{e o} \sqrt{1-\left(\frac{\eta-y_{e o}}{b_{e o}}\right)^{2}}}^{x_{e o}+a_{e o}} \sqrt{1-\left(\frac{\eta-y_{e o}}{b_{e o}}\right)^{2}}\left[\frac{\partial w}{\partial t}\right)^{2}+\frac{h^{3}}{12}\left(\frac{\partial \psi_{\xi}}{\partial t}\right)^{2}+\frac{h^{3}}{12}\left(\frac{\partial \psi_{\eta}}{\partial t}\right)^{2}\right] \mathrm{d} \xi \mathrm{d} \eta
\end{aligned}
$$

If one introduces $x_{e o}=y_{e o}$ in the Eq. (10) and (11), strain and kinetic energies of circular opening is obtained. Similarly as in previous case, in Fig. $3 x_{e o}$ and $y_{e o}$ denote longitudinal and transverse coordinate of elliptic opening centre of gravity, while and $a_{e o}$ and $b_{e o}$ are opening major and minor semi-axis, respectively.

\section{Energy of oval opening}

Oval opening, which often appears in ships and offshore structures, can be treated as a combination of rectangular and circular openings, Fig. 4., where $x_{o \mathrm{o}}$ and $y o_{e o}$ are longitudinal and transverse coordinate of oval opening centre of gravity, while and $a_{o o}$ and $b_{o o}$ are equal to one half of its length and breadth, respectively. 
In that way its strain and kinetic energies can be written in following form:

$$
\begin{aligned}
& T_{o o}=T_{c o 1}+T_{r o}+T_{c o 2} \\
& V_{o o}=V_{c o 1}+V_{r o}+V_{c o 2}
\end{aligned}
$$

and can be calculated by above listed formulae.

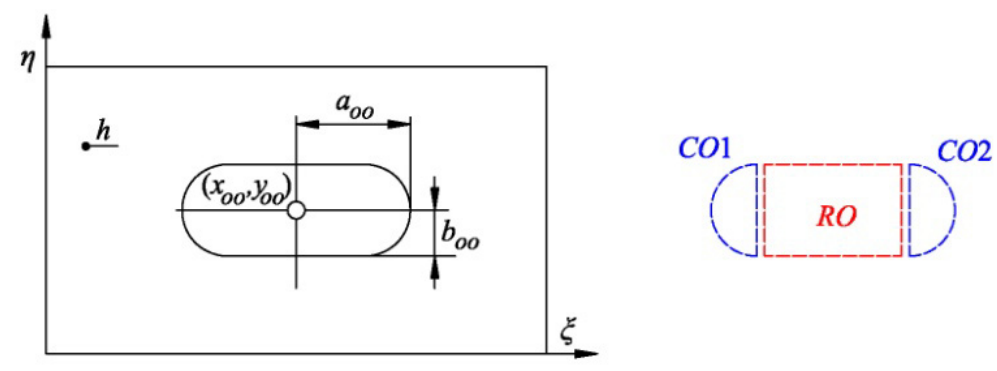

Fig. 4 Rectangular plate with oval opening.

If one wants to apply developed procedure for more than one opening, opening strain and kinetic energies have to be calculated separately, and subtracted from the corresponding energies of plate without openings.

\section{Outline of assumed mode method}

In the assumed mode method, lateral displacement and the rotational angles are expressed by superposing products of orthogonal polynomials:

$$
\begin{aligned}
& w(\xi, \eta, t)=\sum_{m=1}^{M} \sum_{n=1}^{N} a_{m n}(t) X_{m}(\xi) Y_{n}(\eta) \\
& \psi_{\xi}(\xi, \eta, t)=\sum_{m=1}^{M} \sum_{n=1}^{N} b_{m n}(t) \Theta_{m}(\xi) Y_{n}(\eta) \\
& \psi_{\eta}(\xi, \eta, t)=\sum_{m=1}^{M} \sum_{n=1}^{N} c_{m n}(t) X_{m}(\xi) \Phi_{n}(\eta)
\end{aligned}
$$

where $X_{m}(\xi), Y_{n}(\eta), \Theta_{m}(\xi)$ and $\Phi_{n}(\eta)$ are the orthogonal polynomials satisfying the specified elastic edge constraints with respect to $\xi$ and $\eta$. Furthermore, $a_{m n}(t), b_{m n}(t)$ and $c_{m n}(t)$ are the influence coefficients of orthogonal polynomials. Also, $M$ and $N$ are the number of orthogonal polynomials used for approximate solution in $\xi$ and $\eta$ direction, respectively. Eqs. (14), (15) and (16) can be alternatively written in matrix form:

$$
\{z(\xi, \eta, t)\}=[H(\xi, \eta)]\{q(t)\}
$$

where

$$
\{z(\xi, \eta, t)\}=\left\{w(\xi, \eta, t), \psi_{\xi}(\xi, \eta, t), \psi_{\eta}(\xi, \eta, t)\right\}^{T}
$$




$$
\begin{aligned}
& {[H(\xi, \eta)]=\left[\begin{array}{ccccc}
X_{1} Y_{1} \ldots X_{M} Y_{N} & 0 \ldots 0 & 0 \ldots 0 \\
0 \ldots 0 & \Theta_{1} Y_{1} \ldots \Theta_{M} Y_{N} & 0 \ldots 0 \\
0 \ldots & 0 \ldots & \ldots & X_{1} \Phi_{1} \ldots X_{M} \Phi_{N}
\end{array}\right]} \\
& \{q(t)\}=\left\{\begin{array}{llllllllll}
a_{11} & \ldots & a_{M N} & b_{11} & \ldots & b_{M N} & c_{11} & \ldots & c_{M N}
\end{array}\right\}^{T}
\end{aligned}
$$

By substituting the Eq. (4) and (5) into Lagrange's equation of motion below

$$
\frac{\mathrm{d}}{\mathrm{d} t}\left(\frac{\partial T}{\partial \dot{q}_{i}}\right)-\frac{\partial T}{\partial q_{i}}+\frac{\partial V}{\partial q_{i}}=0,
$$

the discrete matrix equation with $3 \times M \times N$ degrees of freedom can be obtained as the following equation:

$$
[M]\left\{\frac{\partial^{2} q(t)}{\partial t^{2}}\right\}+[K]\{q(t)\}=0,
$$

where $[\mathrm{M}]$ and $[\mathrm{K}]$ are the mass and the stiffness matrix, respectively, and their constitution is described in details in Kim et al. (2012).

If we assume harmonic vibrations, i.e.

$$
\{q(t)\}=\{Q\} e^{j \omega t}, \quad w(\xi, \eta, t)=W(\xi, \eta) e^{j \omega t}, \quad \psi_{\xi}(\xi, \eta, t)=\Psi_{\xi}(\xi, \eta) e^{j \omega t}, \quad \psi_{\eta}(\xi, \eta, t)=\Psi_{\eta}(\xi, \eta) e^{j \omega t}
$$

Eq. (22) leads to an eigenvalue problem which gives natural frequencies and eigenvectors of the system, where $j$ is the unit imaginary and $\omega$ represents the angular frequency. The mode shape corresponding to each natural frequency is obtained from the following equation:

$$
\left\{W(\xi, \eta), \Psi_{\xi}(\xi, \eta), \Psi_{\eta}(\xi, \eta)\right\}_{l}^{T}=[H(\xi, \eta)]\{Q\}_{l}
$$

where $l$ represents the order of mode. It should be noted here that when using the assumed mode method, the orthogonal polynomials corresponding to the property of target model should be applied to achieve accurate analysis. For this purpose, the characteristic orthogonal polynomials having the property of Timoshenko beam functions which satisfies the specified edge constraints are used, and their complete derivation is presented by Kim et al. (2012).

In addition, it should be mentioned that developed procedure is not fully physical consistent, since in the Rayleigh-Ritz method all assumed shape function should satisfy at least geometric boundary conditions (Szilard, 2004), which is not unconditionally the case in the presented procedure at the opening boundaries. Namely, it is necessary to say that there exists differrence in real boundary conditions at the free opening edge and remained after excluding that part. In the former case boundary forces are zero, while in the latter case they are equal to the sectional forces. However, if opening dimensions are not too large comparing the plate dimensions, the procedure should normally give quite accurate and reliable results.

\section{NUMERICAL EXAMPLES}

In order to illustrate applicability of the developed procedure, natural vibration analyses of plates with different openings are carried out. Just for the illustration purposes, first, a rectangular plate without openings as a very simple case with available analytical solution is analysed. Then, rectangular plate with single central rectangular opening is analysed. Further on, the procedure is applied to rectangular plate with central elliptic opening. Also, plate with two elliptic openings is analysed as well as a rectangular plate with central circular opening. Finally, results of vibration analysis of a rectangular plate with oval opening, 
which is typical in naval architecture, are presented. The number of polynomials has been set to 13 in all examples, for both $\xi$ and $\eta$ directions, respectively, after convergence test. The values of Young's modulus, material density and Poisson's ratio are the same in all numerical examples and their values are set to $2.1 \times 10^{11} \mathrm{~N} / \mathrm{m}^{2}, 7850 \mathrm{~kg} / \mathrm{m}^{3}$ and 0.3 , respectively. Furthermore, the value of shear factor $k$ is adjusted to 0.86667 in all calculations. In all numerical examples, the results obtained by the proposed procedure are denoted with PS (Present Solution).

\section{Rectangular plate without openings}

A numerical calculation of the natural frequencies has been carried out for a plate without openings, simply supported on the longitudinal edges and clamped on the transversal ones SCSC (simple-clamped-simple-clamped), according to the procedure described in previous section. In Table 1 , the frequency parameter $\lambda$ is presented for two different relative thickness ratios $h / b$, which include thin and moderately thick plate. Non-dimensional frequency parameter is calculated according to following formula:

$$
\lambda=\frac{\omega b^{2}}{\pi^{2}} \sqrt{\frac{\rho h}{D}}
$$

where the value of natural frequency $\omega$ is given in $\mathrm{rad} / \mathrm{s}$. In this case the obtained results are compared with recently presented analytical solutions (Xing and Liu, 2009; Senjanović et al., 2013), as well as with numerical results obtained by finite element method (MSC, 2010). Generally, it is obvious from the shown results, Table 1, that the developed procedure provides very good results comparing to the analytical solution.

Table 1 Frequency parameter $\lambda$ of rectangular plate without openings, SCSC edge constraints.

\begin{tabular}{|c|c|c|c|c|c|c|c|c|}
\hline \multirow{2}{*}{$a / b$} & \multirow{2}{*}{$h / b$} & \multirow{2}{*}{ Method } & \multicolumn{6}{|c|}{ Mode no. } \\
\hline & & & 1 & 2 & 3 & 4 & 5 & 6 \\
\hline \multirow{8}{*}{0.4} & \multirow{4}{*}{0.01} & Xing and Liu (2009) & 14.67 & 16.60 & 20.37 & 26.28 & 34.40 & 39.39 \\
\hline & & Senjanović et al. (2013) & 14.67 & 16.60 & 20.37 & 26.28 & 34.40 & 39.39 \\
\hline & & FEM & 14.62 & 16.38 & 19.90 & 25.52 & 33.34 & 39.26 \\
\hline & & PS & 14.67 & 16.61 & 20.37 & 26.28 & 34.40 & 39.43 \\
\hline & \multirow{4}{*}{0.1} & Xing and Liu (2009) & 10.58 & 11.74 & 14.19 & 17.95 & 22.67 & 22.78 \\
\hline & & Senjanović et al. (2013) & 10.58 & 11.74 & 14.19 & 17.95 & 22.67 & 22.78 \\
\hline & & FEM & 10.69 & 11.91 & 14.45 & 18.27 & 23.03 & 23.14 \\
\hline & & PS & 10.63 & 11.88 & 14.41 & 18.19 & 22.88 & 23.40 \\
\hline \multirow{8}{*}{0.5} & \multirow{4}{*}{0.01} & Xing and Liu (2009) & 9.62 & 11.69 & 15.78 & 22.08 & 25.57 & 27.89 \\
\hline & & Senjanović et al. (2013) & 9.62 & 11.69 & 15.78 & 22.08 & 25.57 & 27.89 \\
\hline & & FEM & 9.57 & 11.50 & 15.40 & 21.50 & 25.45 & 27.40 \\
\hline & & PS & 9.62 & 11.69 & 15.78 & 22.08 & 25.59 & 27.91 \\
\hline & \multirow{4}{*}{0.1} & Xing and Liu (2009) & 7.59 & 9.03 & 11.95 & 16.19 & 16.85 & 18.12 \\
\hline & & Senjanović et al. (2013) & 7.59 & 9.03 & 11.95 & 16.14 & 16.85 & 18.12 \\
\hline & & FEM & 7.64 & 9.11 & 12.09 & 16.38 & 17.07 & 18.29 \\
\hline & & PS & 7.62 & 9.12 & 12.07 & 16.29 & 17.13 & 18.45 \\
\hline
\end{tabular}




\section{Rectangular plate with rectangular opening}

Rectangular plate with length and breadth $1.0 \mathrm{~m}$ and $0.7 \mathrm{~m}$, respectively, is considered. Dimensions of the central opening are $0.2 \times 0.1 \mathrm{~m}$, and different relative thickness ratios $h / a$ are taken into account. Values of the first 3 natural frequencies, calculated according to the developed procedure together with finite element solutions obtained by MSC/NASTRAN (MSC, 2010), are listed in Table 2. Different combinations of edge constraints, i.e. SSSS (simple-simple-simple-simple), CCCC (clamped-clamped-clamped-clamped) and FCSC (free-clamped-simple-clamped), have been analysed. The agreement of the natural frequencies is quite good, especially bearing in mind the fact that dimensions of an opening are quite large comparing to plate dimensions. Natural modes for some selected cases are presented in Figs. 5 and 6, where also good agreement is obvious.

Table 2 Natural frequency $f(H z)$ of rectangular plate with central rectangular opening.

\begin{tabular}{|c|c|c|c|c|c|c|c|c|c|c|}
\hline \multirow{3}{*}{$\begin{array}{c}\text { Mode } \\
\text { no. }\end{array}$} & \multirow{3}{*}{$h / a$} & \multicolumn{9}{|c|}{ Edge constraints } \\
\hline & & \multicolumn{3}{|c|}{ SSSS } & \multicolumn{3}{|c|}{$\mathrm{CCCC}$} & \multicolumn{3}{|c|}{ FCSC } \\
\hline & & PS & FEM & Diff., \% & PS & FEM & Diff., $\%$ & PS & FEM & Diff., \% \\
\hline \multirow{3}{*}{1} & 0.01 & 73.54 & 70.99 & 3.59 & 142.20 & 138.87 & 2.40 & 61.45 & 61.05 & 0.66 \\
\hline & 0.05 & 363.19 & 343.48 & 5.74 & 683.72 & 669.43 & 2.13 & 300.47 & 298.43 & 0.68 \\
\hline & 0.1 & 701.08 & 653.65 & 7.26 & 1237.17 & 1223.66 & 1.10 & 567.56 & 565.48 & 0.37 \\
\hline \multirow{3}{*}{2} & 0.01 & 148.32 & 146.22 & 1.44 & 224.02 & 220.56 & 1.57 & 124.33 & 119.61 & 3.95 \\
\hline & 0.05 & 722.05 & 701.19 & 2.97 & 1055.39 & 1042.54 & 1.23 & 599.11 & 575.86 & 4.04 \\
\hline & 0.1 & 1343.37 & 1297.22 & 3.56 & 1828.69 & 1830.19 & -0.08 & 1101.90 & 1070.89 & 2.90 \\
\hline \multirow{3}{*}{3} & 0.01 & 224.70 & 213.90 & 5.05 & 337.09 & 312.84 & 7.75 & 159.41 & 158.26 & 0.73 \\
\hline & 0.05 & 1076.00 & 1007.36 & 6.81 & 1537.13 & 1415.95 & 8.56 & 763.27 & 759.25 & 0.53 \\
\hline & 0.1 & 1928.30 & 1795.46 & 7.40 & 2499.41 & 2346.04 & 6.54 & 1368.01 & 1372.45 & -0.32 \\
\hline
\end{tabular}

PS
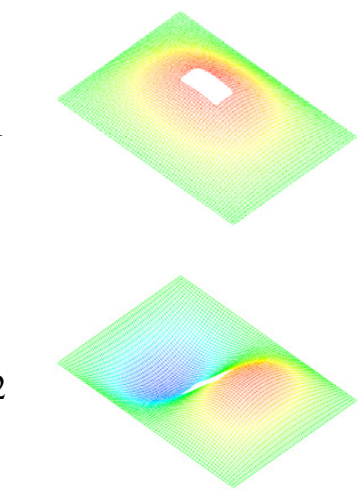

3
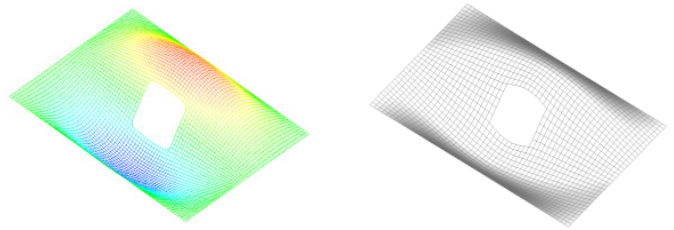

Fig. 5 Mode shapes of rectangular plate with central rectangular opening, $h=0.01 \mathrm{~m}$, SSSS.
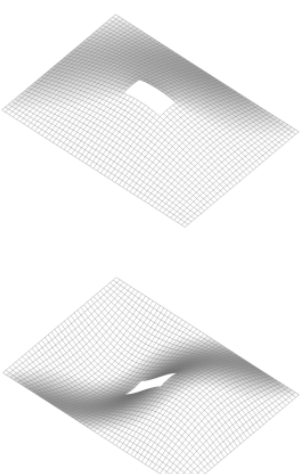

PS

FEM

2
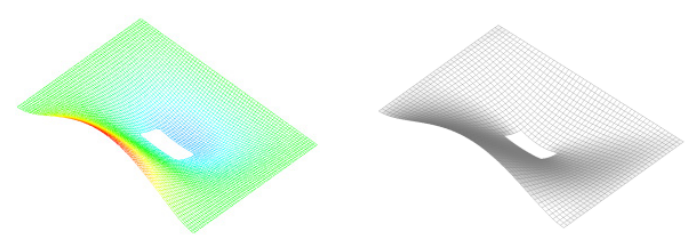

3
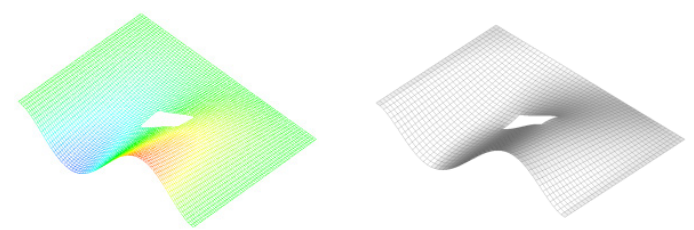

Fig. 6 Mode shapes of rectangular plate with central rectangular opening, $h=0.01 m$, FCSC. 


\section{Rectangular plate with elliptic openings}

Natural vibration analysis of rectangular plate of same dimensions as in previous case, with central elliptic opening and two elliptic openings at arbitrary defined locations has been also performed. In the former case, the dimensions of the opening are $a_{e o}=0.2 \mathrm{~m}$ and $b_{e o}=0.1 \mathrm{~m}$, while in the latter one $a_{e o}=0.08 \mathrm{~m}$ and $b_{e o}=0.15 \mathrm{~m}$. Due to reason of simplicity, only FSCS case is analysed. In this case also reasonable agreement between both, natural frequencies listed in Table 3 , and natural modes shown in Figs. 7 and 8, is achieved.

Table 3 Natural frequencies $f(H z)$ of rectangular plate with elliptic openings.

\begin{tabular}{|c|c|c|c|c|c|c|c|}
\hline \multirow{2}{*}{$\begin{array}{c}\text { Mode } \\
\text { no. }\end{array}$} & \multirow{2}{*}{$h / a$} & \multicolumn{3}{|c|}{1 Opening } & \multicolumn{3}{c|}{ 2 Openings } \\
\cline { 3 - 8 } & & PS & FEM & Diff., $\%$ & PS & FEM & Diff., $\%$ \\
\hline \multirow{2}{*}{1} & 0.01 & 40.74 & 39.79 & 2.39 & 39.86 & 39.29 & 1.45 \\
\cline { 2 - 8 } & 0.05 & 197.28 & 192.81 & 2.32 & 195.68 & 190.07 & 2.95 \\
\hline \multirow{2}{*}{2} & 0.01 & 108.17 & 107.54 & 0.59 & 108.62 & 105.70 & 2.76 \\
\cline { 2 - 8 } & 0.05 & 527.31 & 523.31 & 0.76 & 528.26 & 512.50 & 3.08 \\
\hline \multirow{2}{*}{3} & 0.01 & 139.64 & 136.09 & 2.61 & 141.70 & 137.35 & 3.17 \\
\cline { 2 - 8 } & 0.05 & 670.99 & 655.29 & 2.40 & 681.12 & 659.46 & 3.28 \\
\hline
\end{tabular}

PS

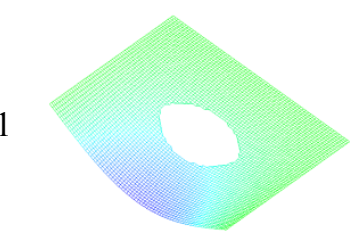

2

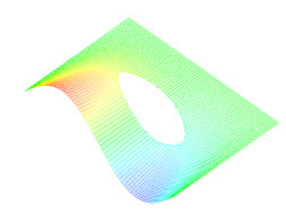

3

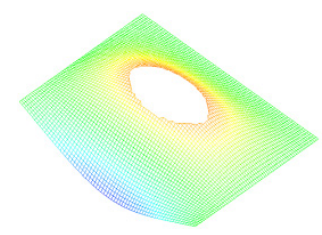

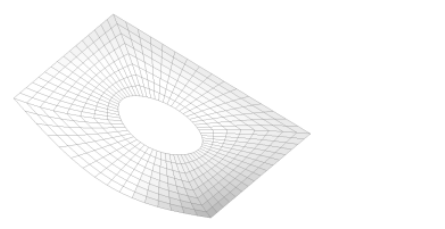
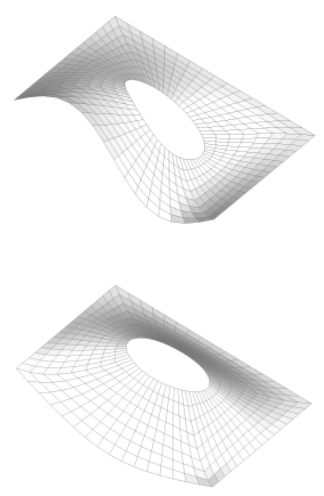

Fig. 7 Mode shapes of rectangular plate with central elliptic opening, $h=0.05 \mathrm{~m}$, FSCS.

PS

FEM
2

1

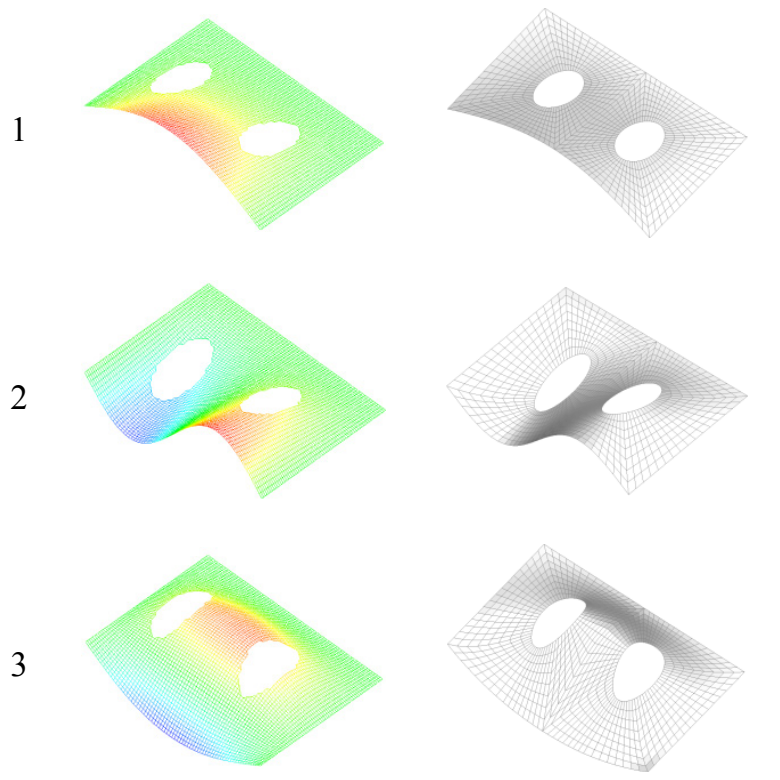

Fig. 8 Mode shapes of rectangular plate with two elliptic openings, $h=0.05 m$, FSCS.

\section{Rectangular plate with circular opening}

Mathematical model for vibration analysis of rectangular plates with circular openings of radius $R$ represents a special case of a plate with elliptic opening, and that means the above formulae for case of elliptic opening are valid if $a_{e o}=b_{b o}=R$. In this case the obtained results are compared to those presented by Grossi et al. (1997), where natural frequencies, obtained by optimized Rayleigh-Ritz method, are converted in dimensionless frequency parameters. The comparison with FEM results is 
also provided. Non-dimensional frequency parameters $\Omega_{i j}$ obtained for a rectangular plate with different length to breadth, $a / b$, and diameter to length ratios, $\eta=2 R / a$, respectively, are calculated according to following expression:

$$
\Omega_{i j}=\omega_{i j} a^{2} \sqrt{\frac{\rho h}{D}},
$$

and shown in Tables 4 and 5, for different combinations of classical boundary conditions, where differences in \% between FEM and other results are given in parentheses.

Table 4 Frequency parameter $\Omega_{i j}$ and its difference in $\%$ between FEM and other method of rectangular plate with circular opening, CCCC edge constraints.

\begin{tabular}{|c|c|c|c|c|c|}
\hline \multirow{3}{*}{$a / b$} & \multirow{3}{*}{ Method } & \multicolumn{4}{|c|}{$\eta=2 R / a$} \\
\hline & & \multicolumn{2}{|c|}{0.2} & \multicolumn{2}{|c|}{0.3} \\
\hline & & $\Omega_{11}$ & $\Omega_{22}$ & $\Omega_{11}$ & $\Omega_{22}$ \\
\hline \multirow{3}{*}{1} & FEM & 35.93 & 104.13 & 37.90 & 96.41 \\
\hline & Grossi et al. (1997) & $37.85(5.34 \%)$ & $106.74(2.51 \%)$ & $40.78(7.60 \%)$ & $105.74(9.68 \%)$ \\
\hline & PS & $36.72(2.20 \%)$ & $105.37(1.19 \%)$ & $39.34(3.80 \%)$ & $103.45(7.30 \%)$ \\
\hline \multirow{3}{*}{1.5} & FEM & 62.40 & 170.66 & 71.26 & 167.88 \\
\hline & Grossi et al. (1997) & $65.82(5.48 \%)$ & $177.76(4.16 \%)$ & $74.72(4.86 \%)$ & $177.58(5.78 \%)$ \\
\hline & PS & $63.77(2.20 \%)$ & $175.12(2.61 \%)$ & $72.15(1.25 \%)$ & $171.58(2.20 \%)$ \\
\hline \multirow{3}{*}{2} & FEM & 105.02 & 273.51 & 117.10 & 267.65 \\
\hline & Grossi et al. (1997) & $109.76(4.49 \%)$ & $284.24(3.92 \%)$ & $129.41(10.51 \%)$ & $286.56(7.07 \%)$ \\
\hline & PS & $106.38(1.29 \%)$ & $278.24(1.73 \%)$ & $118.15(0.90 \%)$ & $274.12(2.42 \%)$ \\
\hline
\end{tabular}

Table 5 Frequency parameter $\Omega_{i j}$ and its difference in \% between FEM and other method of rectangular plate with circular opening, CSCS edge constraints.

\begin{tabular}{|c|c|c|c|c|c|}
\hline \multirow{3}{*}{$a / b$} & \multirow{2}{*}{ Method } & \multicolumn{4}{|c|}{$\eta=2 R / a$} \\
\cline { 3 - 6 } & & & \multicolumn{2}{|c|}{0.3} \\
\cline { 3 - 6 } & & $\Omega_{11}$ & $\Omega_{22}$ & $\Omega_{11}$ & $\Omega_{22}$ \\
\hline \multirow{3}{*}{1} & FEM & 28.65 & 91.38 & 29.43 & 85.24 \\
\cline { 2 - 6 } & Grossi et al. (1997) & $30.10(5.06 \%)$ & $93.58(2.41 \%)$ & $31.83(8.15 \%)$ & $92.67(8.72 \%)$ \\
\cline { 2 - 6 } & PS & $29.22(1.99 \%)$ & $92.35(1.06 \%)$ & $30.49(3.60 \%)$ & $90.74(6.45 \%)$ \\
\hline \multirow{3}{*}{1.5} & FEM & 57.38 & 162.66 & 63.42 & 160.70 \\
\cline { 2 - 6 } & Grossi et al. (1997) & $60.06(4.67 \%)$ & $169.44(4.17 \%)$ & $65.93(3.96 \%)$ & $169.10(5.23 \%)$ \\
\hline \multirow{2}{*}{2} & PS & $58.49(1.93 \%)$ & $166.63(2.44 \%)$ & $64.02(0.95 \%)$ & $163.99(2.05 \%)$ \\
\cline { 2 - 6 } & Grossi et al. (1997) & $103.90(3.53 \%)$ & $278.40(3.83 \%)$ & $117.32(8.26 \%)$ & $279.76(6.32 \%)$ \\
\cline { 2 - 6 } & PS & $101.44(1.08 \%)$ & $272.45(1.61 \%)$ & $109.22(0.78 \%)$ & $269.44(2.39 \%)$ \\
\hline
\end{tabular}


Comparison of non-dimensional vibration parameters for rectangular plate with circular openings, Tables 4 and 5, actually shows that developed procedure as well as optimized Rayleigh-Ritz method (Grossi et al., 1997) overestimate natural frequencies. However, comparing to optimized Rayleigh-Ritz method which is more complex, developed procedure gives closer results to those obtained by finite element method, which may be considered as the most accurate ones. Also, comparison of natural modes in this case, Fig. 9, shows good agreement.

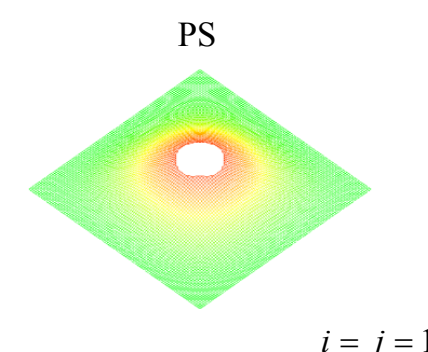

$$
i=j=1
$$

FEM

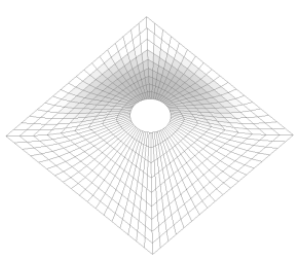

Fig. 9 Mode shapes of rectar

\section{Rectangular plate with oval opening}

Finally, natural vibration analysis of rectangular plate with oval opening has been performed. It should be noted that oval

Table 6 Natural frequencies $f(\mathrm{~Hz})$ of rectangular plate with oval opening.

\begin{tabular}{|c|c|c|c|c|c|c|c|}
\hline \multirow{2}{*}{$\begin{array}{c}\text { Mode } \\
\text { no. }\end{array}$} & $h(m)$ & \multicolumn{3}{|c|}{ FCSC } & \multicolumn{3}{c|}{ CFCC } \\
\cline { 2 - 7 } & & PS & FEM & Diff., $\%$ & PS & FEM & Diff., $\%$ \\
\hline \multirow{2}{*}{1} & 0.01 & 125.51 & 124.44 & 0.86 & 229.73 & 227.54 & 0.96 \\
\cline { 2 - 7 } & 0.05 & 601.51 & 597.88 & 0.61 & 1072.46 & 1069.06 & 0.32 \\
\hline \multirow{2}{*}{2} & 0.01 & 245.46 & 233.50 & 5.12 & 309.48 & 301.80 & 2.54 \\
\cline { 2 - 8 } & 0.05 & 1144.54 & 1098.48 & 4.19 & 1420.01 & 1403.00 & 1.21 \\
\hline \multirow{2}{*}{3} & 0.01 & 322.93 & 319.30 & 1.14 & 473.11 & 456.56 & 3.62 \\
\cline { 2 - 8 } & 0.05 & 1489.45 & 1478.12 & 0.77 & 2089.39 & 2064.10 & 1.23 \\
\hline
\end{tabular}

PS

FEM

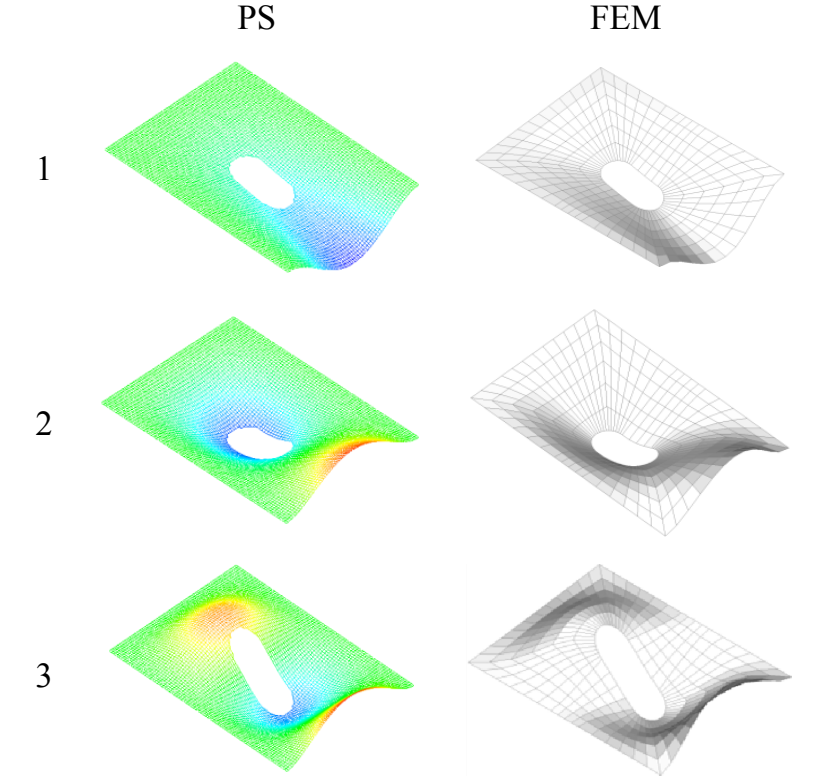

Fig. 10 Mode shapes of rectangular plate with oval opening, $h=0.05 m$, CFCC. 
type opening is widely adopted in the ship and offshore structures, but at the same time its FE modelling is rather time-consuming task. In that sense, application of the presented method to this problem is advantageous.

Plate length and breadth yield $0.7 \mathrm{~m}$ and $0.5 \mathrm{~m}$, respectively, while oval opening dimensions are $a_{o o}=0.1 \mathrm{~m}$ and $b_{o o}=0.05 \mathrm{~m}$. First three natural frequencies of rectangular plate with oval opening, for different relative thickness and boundary conditions, respectively, are shown in Table 6. Corresponding mode shapes for a selected case are presented in Fig. 10. Similarly as in previous cases, acceptable agreement of the results is achieved.

\section{Influence of opening size on plate dynamic response}

Influence of the opening size on plate natural frequencies is considered analyzing natural vibrations of rectangular plate with length and breadth $2.0 \mathrm{~m}$ and $1.0 \mathrm{~m}$, respectively, and plate thickness equal to $0.03 \mathrm{~m}$. Due to reason of simplicity, CCCC boundary conditions are applied, and ratios of opening and plate areas, $A_{o}$, and, $A_{p}$, respectively, has been varied from zero to 0.20. As expected, the best agreement of the result is achieved in case of plate without openings, Fig. 11. Although the difference increases with the opening increase, it still remains acceptable for larger opening size.

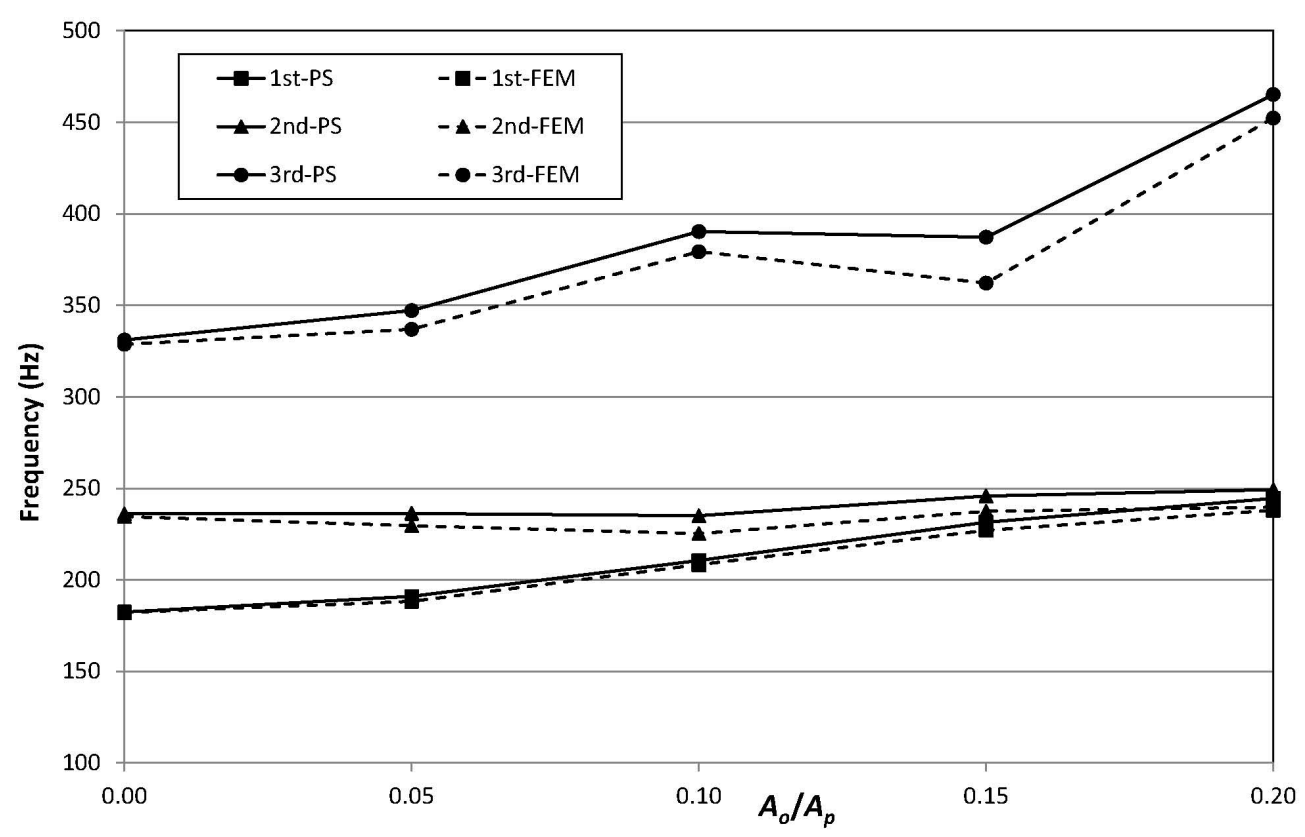

Fig. 11 Natural frequencies of a plate with different sizes of rectangular opening, CCCC.

Concerning the opening size influence on natural frequency, it differs from one natural mode to another. One can notice that natural frequency of the fundamental mode slightly increases, while the second doesn't changes too much. Third natural mode is particularly interesting when increasing $A_{o} / A_{p}$ ratio from 0.1 to 0.15 , where natural frequency doesn't increase. This effect is actually a result of mode shape change as can be seen in Fig. 12.
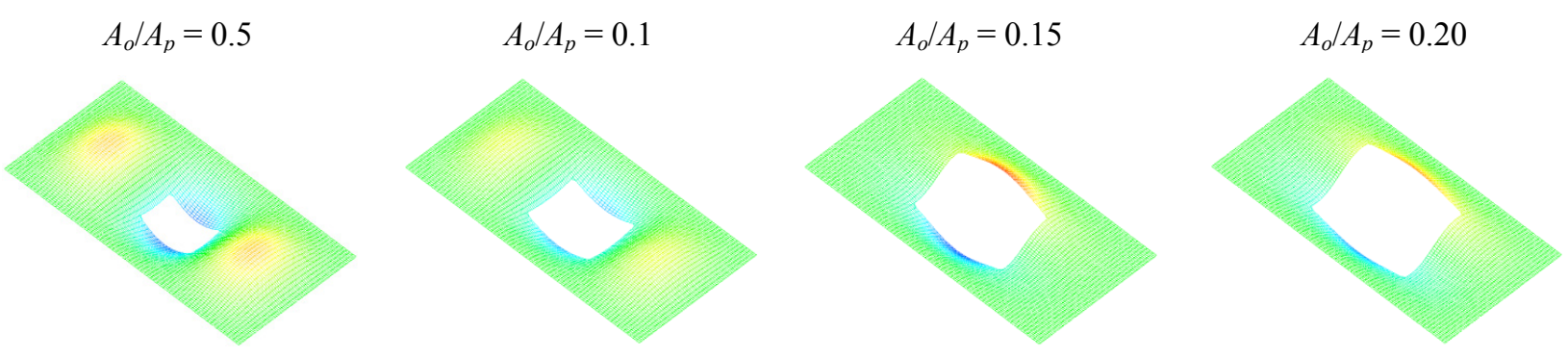

Fig. 12 Third natural mode of a plate with different sizes of rectangular opening, CCCC. 


\section{CONCLUSION}

An approximate procedure for natural vibration analysis of rectangular plates with openings and arbitrary edge constraints is presented. The opening effect is accounted for in a very simple and intuitive way, i.e. by subtracting its strain and kinetic energies from the corresponding total plate energies. Numerical examples, which include analysis of natural vibrations of rectangular plates with variable thicknesses and with different opening shapes (rectangular, elliptic, circular, oval) and sizes show very good agreement with the results obtained by FEM as well as to some results that are presented in the relevant literature. Moreover, despite its simplicity, the presented procedure can give even closer results to FEM comparing to some more complex methods, as shown in the case of rectangular plate with circular opening. Anyhow, it should be mentioned that the procedure should be applied to plates with smaller openings comparing to plate dimensions, as for instance to vibration analysis of swash bulkheads with small openings etc. Thus, it can be used as a reliable alternative to widely used FEM, which may require much more time in model preparation, which is especially pronounced in case of rectangular plate with oval opening. Future investigations should be focused on the application of assumed mode method to vibration analysis of more complex structural members of ships and offshore structures, as for instance stiffened panels, stiffened panels with openings of different shape, rectangular plates with openings or stiffened panels partially or fully immersed into the water, etc.

\section{ACKNOWLEDGEMENTS}

This work was supported by the National Research Foundation of Korea (NRF), grant funded by the Korean Government (MSIP) through GCRC-SOP (Grant No. 2011-0030013).

\section{REFERENCES}

Aksu, G. and Ali, R., 1976. Determination of dynamic characteristics of rectangular plates with cut-outs using a finite difference formulation. Journal of Sound and Vibration, 44, pp.147-158.

Ali, R. and Atwal, S.J., 1980. Prediction of natural frequencies of rectangular plates with circular cutouts. Computers and Structures, 12(6), pp.819-823.

Bathe, K.J., Wilson, E.L. and Peterson, F.E., 1973. A structural analysis program for static and dynamic response of linear systems. Report No. EERC 73-11, University of California, Berkeley, California.

Chung, J.H., Chung, T.Y. and Kim, K.C., 1993. Vibration analysis of orthotropic Mindlin plates with edges elastically restrained against rotation. Journal of Sound and Vibration, 163(1), pp.151-163.

Grossi, R.O., del V. Arenas B. and Laura, P.A.A., 1997. Free vibration of rectangular plates with circular openings. Ocean Engineering, 24(1), pp.19-24.

Gutierrez, R.H., Laura, P.A.A. and Pombo, J.L., 1987. Higher frequencies of transverse vibration of rectangular plates elastically restrained against rotation at the edges with a central free hole. Journal of Sound and Vibration, 117(1), pp.202206.

Hegarty, R.F. and Ariman, T., 1975. Elasto-dynamic analysis of rectangular plates with circular holes. International Journal of Solids and Structures, 11(7-8), pp.895-906.

Huang, M. and Sakiyama, T., 1998. Free vibration analysis of rectangular plates with variable thickness. Journal of Sound and Vibration, 216(3), pp.379-397.

Huang, M. and Sakiyama, T., 1999. Free vibration analysis of rectangular plates with variously-shaped holes. Journal of Sound and Vibration, 226(4), pp.769-786.

Kim, K., Kim, B.H., Choi, T.M. and Cho, D.S., 2012. Free vibration analysis of rectangular plate with arbitrary edge constraints using characteristic orthogonal polynomials in assumed mode method. International Journal of Naval Architecture and Ocean Engineering, 4(3), pp.267-280.

Kwak, M.K. and Han, S., 2007. Free vibration analysis of rectangular plate with a hole by means of independent coordinate coupling method. Journal of Sound and Vibration, 306(1-2), pp.12-30.

Laura, P.A.A., Gutierrez, R.H., Ercoli, L., Utjes, J.C. and Carnicer, R., 1987. Free vibration of rectangular plates elastically restrained against rotation with circular or square free openings. Ocean Engineering, 14(4), pp.285-293. 
Leissa, A.W., 1969. Vibration of plates, NASA Report No. SP-160. Scientific and Technical Information Division, National Aeronautics and Space Administration.

Mindlin, R.D., Schacknow, A. and Deresiewicz, H., 1956. Flexural vibrations of rectangular plates. Journal of Applied Mechanics, 23, pp.430-436.

Monahan, L.J., Nemergut, P.J. and Maddux, G.E., 1970. Natural frequencies and mode shapes of plates with interior cutouts. The Shock and Vibration Bulletin, 41(3), pp.37-49.

MSC, 2010. MD Nastran 2010 Dynamic analysis user's guide. MSC Software.

Paramasivam, P., 1973. Free vibration of square plates with square openings. Journal of Sound and Vibration, 30(2), pp.173-178.

Reddy, J.N., 1982. Large amplitude flexural vibration of layered composite plates with cutouts. Journal of Sound and Vibration, 83(1), pp.1-10.

Senjanović, I., Vladimir, N. and Tomić, M., 2013. An advanced theory of moderately thick plate vibrations. Journal of Sound and Vibration, 332(7), pp.1868-1880.

Szilard, R., 2004. Theories and applications of plate analysis. John Wiley \& Sons.

Xing, Y. and Liu, B., 2009. Characteristic equations and closed-form solution for free vibrations of rectangular Mindlin plates. Acta Mechanica Solida Sinica, 22(2), pp.125-136. 